\title{
Sistema de Apoio à Decisão por PLN para consultas de Pragas na Cultura da Soja
}

\author{
Carolinne Roque e Faria ${ }^{1}$, Maurilio Martins Campano Junior ${ }^{1}$ \\ Cinthyan R. Sachs C. de Barbosa ${ }^{1}$, \\ ${ }^{1}$ Programa de Pós-Graduação em Ciência da Computação - Universidade Estadual de \\ Londrina (UEL) - 86057-970 - Londrina, PR - Brazil \\ carolinne.rf@outlook.com,maurilio.campanojr@gmail.com, cinthyan@uel.br
}

\begin{abstract}
The volumes of agricultural data available are increasing. One of the obstacles faced in this context is to approach the data in Natural Language (NL) for information management and process improvements of a culture. This paper proposes the development of a Decision Support System for NL processing in the field of agronomy, using the data on the pests in the soybean crop that will be stored in a database, thus allowing consultations to build dialogues and obtain accurate diagnoses. The proposed theme indicated possibilities of positive effects and that may facilitate the work of agricultural professionals who need to be involved with a large volume of information.
\end{abstract}

Resumo. Os volumes de dados agrícolas disponíveis são cada vez maiores. Uma dos obstáculos enfrentados nesse contexto é abordar os dados em Linguagem Natural (LN) para gestão das informações e melhorias de processos de uma cultura. Este artigo propõe o desenvolvimento de um Sistema de Apoio à Decisão por processamento da LN para o campo da agronomia, utilizando os dados sobre as pragas na cultura da soja que serão armazenados em um banco de dados, permitindo assim consultas para construir diálogos e obter diagnósticos precisos. O tema proposto indicou possibilidades de efeitos positivos e que pode facilitar o trabalho de profissionais da agricultura que precisam se envolver com um amplo volume de informações.

\section{Introdução}

O grande avanço científico e tecnológico alcançado na atualidade dentro do segmento agrícola passa pelas necessidades do produtor rural de aumentar a produtividade e diminuir as perdas no campo de maneira eficiente, por meio da utilização de tecnologias da informação.

Uma das principais bases da economia de diversos países é a agricultura, principalmente no Brasil [Hiraoka e Jacopini 2018], que nesse setor tem seu potencial reconhecido globalmente. Contudo, os prejuízos que afetam a produtividade das lavouras deve-se às pragas. Pesquisas feitas em 2015 [Agrolink 2015], indicam que da produção média anual brasileira, cerca de $7,7 \%$ é perdida por conta de ataques de lagartas, moscas e outras doenças, correspondendo a 25 milhões de toneladas, equivalente a $\mathrm{R} \$ 55$ bilhões. As perdas causadas por ataques de pragas em contexto mundial, chegaram a cerca de 5\% do Produto Interno Bruto (PIB) mundial, semelhante a US\$ 1,4 trilhão. 
A área de Processamento de Linguagem Natural (PLN) [Barbosa 1997, 1998] expõe soluções computacionais que repercutem na compreensão da língua natural (escrita ou falada), como, por exemplo, a Análise Léxico-Morfológica (tokenização), Análise Sintática (parsing), Análise Semântica e Análise Pragmática.

O desfecho de um sistema de PNL provém da compreensão do domínio da aplicação, ou seja, da integridade relativa do modelo de linguagem natural que ele encapsula e da adequação ou eficiência de seus algoritmos [Manaris e Slator 1996]. Um dos motivos que torna o PLN tão importante [Agosti 2003] é que modifica as técnicas de processamento dos computadores o mais próximo possível dos mecanismos do raciocínio humano.

É primordial uma percepção imediata da linguagem da agricultura, possibilitando o emprego por computadores e também que analise os pontos em linguagem natural para que esses contribuam com os estudantes de agronomia e até mesmo com os agricultores nas tomadas de decisões, de acordo com o diagnóstico feito por meio de diálogos com a finalidade de proporcionar consultas produtivas sobre as descrições de diagnósticos e fazer escolhas de acordo com as alternativas geradas.

Este artigo está organizado da seguinte maneira: a seção 2 descreve os trabalhos relacionados à temática do trabalho; a seção 3 apresenta a fundamentação teórica; na seção 4 a proposta de uma abordagem em PLN para consulta à Base de Dados de Pragas na Cultura da Soja é exibida; na seção 5 a metodologia e desenvolvimento são apresentados; por fim na seção 6 tem as considerações finais.

\section{Trabalhos Relacionados}

A área da informática está sendo utilizada para automatizar e melhorar o desempenho de suas tarefas e no caso da agricultura não é diferente. Dentre os trabalhos nessa área tem-se o uso da Internet das Coisas (IoT) [Gu e Jing 2011]. Tal trabalho, por exemplo, propõe aplicações específicas de IoT para medir a qualidade de controlar estritamente as fontes de segurança, aperfeiçoar o monitor dos produtos agrícolas frescos, e assim, construir um sistema de informação de gestão desses produtos para aumentar o nível de integração da cadeia de suprimentos e reduzir os custos de gerenciamento desta para melhorar a eficiência da cadeia de abastecimento.

Um chatbot para a divulgação do Atlas Linguístico do Brasil também foi apresentado em [Moreno et al. 2015], dando assim ao referido projeto, características de interdisciplinaridade na área de Linguística e Ciências da Computação, com o foco principal em Processamento de Linguagem Natural. A ideia com esse chatbot, além de divulgar o Atlas, é despertar em outros pesquisadores o desejo de desenvolver outros assuntos que sejam peculiar ao ensino, utilizando uma ferramenta comum entre os jovens que é o WhatsApp.

O trabalho de Rocha e Sartin (2018), por outro lado, alia a visão computacional à análise de folhas de soja, com o objetivo de encontrar e extrair características que permitam a detecção de doenças foliares. Tal trabalho faz uso de pré-processamento da imagem de folhas de soja, utilizando-se de filtros de média, mediana e métodos de detecção de bordas e linhas para identificar a folha da soja. Utiliza-se também de 
segmentação de imagens, porém com uma dificuldade na obtenção das veias nas folhas de soja mais escuras.

Em Silva (2018) foi realizado um estudo por meio da utilização de metadados e ontologias para a melhoria do processo de intercâmbio de dados para processos de produção da cultura da erva-mate (Ilex Paraguariensis St. Hill.). Uma ontologia de domínio foi uesada, resultando em estruturas de informação para intercâmbio e rastreabilidade de dados agrícolas, melhorando os processos e gestão das informações. Para o processo de desenvolvimento da ontologia foi utilizada a Ferramenta Protégé, aplicando à Metodologia Ontology Development 101.

Já em Franco (2017) foram utilizados de processamento de imagens para controle e identificação do fungo Cercospora kikuchii, causador da mancha púrpura na folha da soja, que pode implicar em prejuízos na produtividade e também na produção dos derivados. O sistema computacional foi desenvolvido utilizando a biblioteca de visão computacional $O p e n C V$, em conjunto com a linguagem Java e integrando com a ferramenta Weka. A análise de 150 sementes sadias e outras 150 sementes contaminadas se mostrou eficiente, com um índice $86 \%$ de precisão.

O aplicativo de Rosa (2018) apresenta uma metodologia para classificação de pragas comuns no estado do Paraná por meio da rede neural convolucional, baseada na arquitetura Inception V3, na qual um sistema computacional do tipo cliente-servidor foi criado a fim de prover a classificação de pragas, totalizando 1000 imagens. A taxa de assertividade da rede neural obteve $92,5 \%$ de assertividade, configurando um excelente resultado.

\section{Fundamentação Teórica}

Técnicas de PLN para o desenvolvimento de uma ferramenta de apoio à decisão que auxilie no processo de ensino de agronomia para o estudo de pragas no cultivo da soja serão apresentadas a seguir.

Analisando os principais produtos agrícolas no contexto econômico mundial e nacional, a soja se destaca. Tal sucesso se dá devido à competitividade do sojicultor no mercado mundial de grãos, que são devido às condições edafoclimáticas brasileiras e a utilização de tecnologias para o aumento da produtividade, embora não haja comprovação científica do benefício das tecnologias [Carvalho et al. 2013].

Além disso, a cultura da soja pode ser atacada por vários tipos de pragas [Santos 1995], [Moreira e Aragão 2009], [Sosa-Gomez 2010] e [Ávila 2011]. A capacidade de identificação das pragas em seus inúmeros períodos de crescimento e as dificuldades enfrentadas a cada colheita foram as principais motivações para este trabalho com o uso de técnicas de PLN, que não foi encontrado trabalho correlato específico para a cultura da soja.

Um sistema de PLN é capaz de disponibilizar módulos autômatos, que realizam tarefas específicas e especializadas, e outros módulos que armazenam um modelo de conhecimento proposicional, que visa a criar simulacros de parcelas de mundo que the servem de referencial para interpretar os enunciados linguísticos [Di Felippo e Dias-daSilva 2006]. Apesar da arquitetura de um sistema de PLN variar de acordo com as especificidades da aplicação, dois grupos de componentes são imprescindíveis para a 
implementação de qualquer sistema desse tipo. Di Felippo e Dias-da-Silva (2006) sugerem as bases de conhecimento e os módulos de processamento que atuam sobre essas bases, como mostrados na Figura 1.

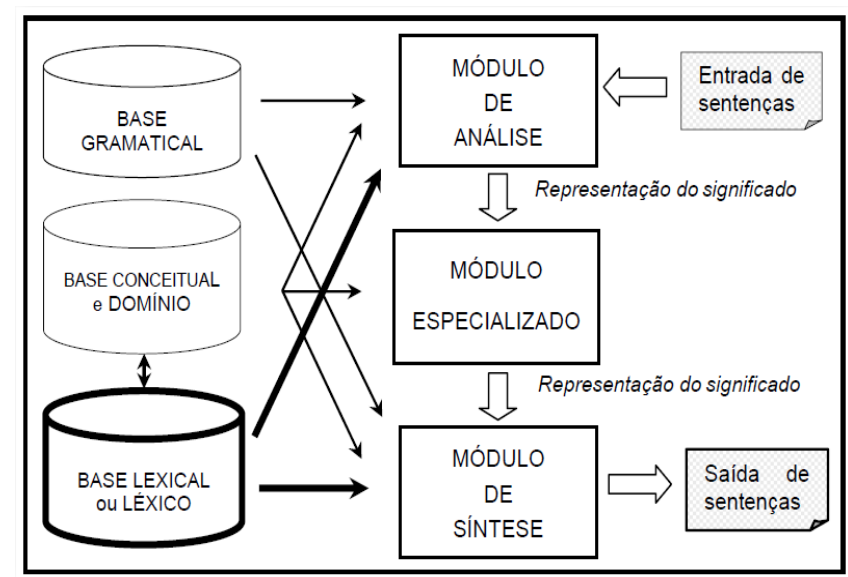

Figura 1. Arquitetura de um sistema de PLN genérico [Di Felippo e Dias da Silva 2006]

A Figura 2 apresenta as cinco fases utilizadas na análise de PLN [Dale 2010], que é dividida em vários segmentos, baseado nos traços linguísticos.

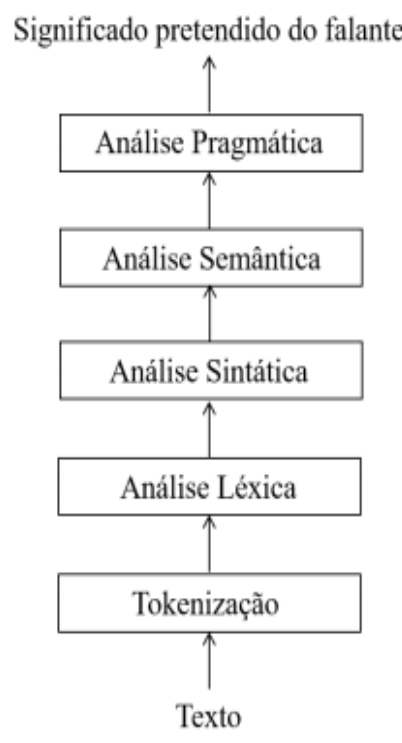

Figura 2 - Estágios de análise no PLN [Dale 2010]

O processo conhecido como segmentação de palavras é chamado de tokenização, reconhecendo letras maiúsculas e minúsculas, palavras compostas e abreviadas, quebra de caracteres, considerando também as ambiguidades lexicais e atenção à verificação ortográfica.

A análise léxica é responsável por manipular o léxico, que é composto por palavras que armazena seu significado e categoria lexical e pode conter, por exemplo, seu radical, que a partir dele podem ser formadas outras palavras. 
A tarefa da análise sintática (parsing) é de extrair informações de uma frase representada por meio de gramáticas e árvores sintática. Maiores detalhes dessas no uso da agricultura serão exibidos na próxima seção.

A análise semântica consiste em analisar os significados das palavras, ou seja, interpretar as expressões fixadas, sentenças inteiras e enunciados no contexto [Goddard and Schalley 2010], pois as palavras/sentenças podem ser ambíguas.

Em relação à análise pragmática, o estudo fundamenta-se em reconhecimento de palavras dentro de um contexto [Muller 2006]. A estrutura não parte de apenas uma frase, visto que busca nas frases do texto para compreender a frase analisada.

A aplicação desse conhecimento em PLN é indispensável, principalmente quando o destaque do processamento interpreta as informações do usuário, permitindo o acesso à base de dados e os são textos esquemáticos, com uma parte variável que só é determinada no decorrer do discurso (ex.: essa, isso, aquela etc.). Isso é estudado na fase de Análise Pragmática mostrada na Figura 2.

A arquitetura de um sistema computacional [Dias-da-Silva et al. 2007] que executa a língua natural pode variar de acordo com as especificidades da aplicação. $O$ tradutor automático é um possível exemplo de aplicação em um sistema mais completo que deverá ser capaz de:

I - Identificar, ou seja, extrair cada uma das palavras da sentença;

II - Analisar sintaticamente a sentença, ou seja, relacionar a cada palavra suas propriedades e funções sintáticas;

III - Construir uma nova sentença para retomar o sentido das informações levantadas anteriormente, ou seja, extrair um significado absoluto da mesma, a partir dos significados das palavras e das relações entre elas;

IV - Associar o significado extraído em uma representação adequada. Essa representação pode ser independente da língua destino (interlíngua).

V - Transformar a representação anterior em uma sentença na língua destino, ou seja, traduzir ou associar as palavras da língua origem para a língua destino, desde que sejam equivalentes.

A partir disso, é possível determinar as inúmeras maneiras de escrever ou expressar um certo material informacional, fazendo com que o sistema controle automaticamente a geração da tarefa e que o processo dessa seja mais simples do que a de interpretação, como sistemas que têm a função específica de transmitir informações constantes em uma base de dados em PLN, ou seja, de sistemas que a missão seja declarar ou informar. Ainda assim, visar a simplificação do sistema pode prejudicar o resultado, dado que o texto implica na modificação do conteúdo informacional e também no domínio dos pontos comunicativos, conforme as intenções do autor do texto, como a tradução automática, que propõe uma correspondência mais verdadeira entre o texto de origem e o texto de destino.

\section{PLN para consultas em BD sobre pragas na cultura da soja}

A arquitetura geral de um sistema de interpretação, baseado em Silva et al. (2007), é exibida na Figura 3, a qual está sendo pautado este artigo. 


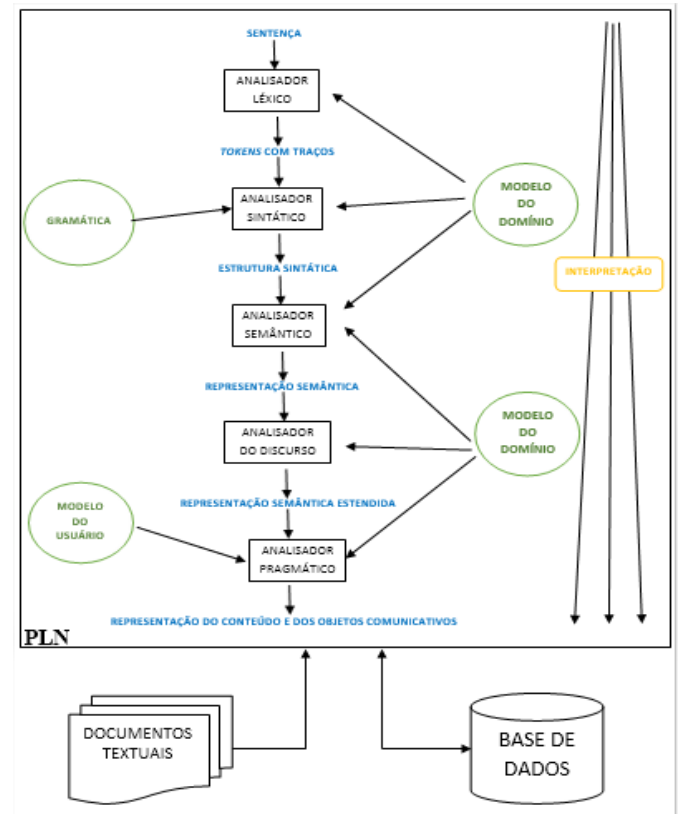

Figura 3 - Arquitetura de um Sistema de Interpretação de Linguagem Natural [Di Felippo e Dias-da-Silva 2006]

Os indicadores de processamento são representados por retângulos, enquanto que os recursos necessários ao processamento, de ordem linguística (gramática, léxico) ou não (modelos do domínio e do usuário), aparecem representados por círculos. Esses recursos são essenciais durante a fase de interpretação, bem como na de geração. Destaca-se as aplicações que não envolvem a interpretação de uma sentença que têm sua arquitetura simplificada, eliminando-se alguns dos módulos e/ou bases de conhecimento que aparecem na Figura 3.

Como este trabalho aqui apresentado não fará, em um primeiro momento, análises semântica e pragmática das perguntas feitas à Base de Dados de Pragas na Cultura da Soja, vamos nos ater as fases Léxica e Sintática, bem como à representação de um dicionário e gramática, a qual será abordada na seção seguinte.

\section{Metodologia e Desenvolvimento}

O método empregado na seleção de palavras foi realizado em três etapas: reunião com agrônomos, leitura e indicações bibliográficas sobre as pragas, e consultas nos site EMBRAPA (Empresa Brasileira de Pesquisas Agropecuária) [Embrapa 2018] e demais manuais de pragas da soja [Santos 1995], [Moreira e Aragão 2009], [Sosa-Gomez 2010] e [Ávila 2011].

Com base no diagnóstico das características das pragas extraídas dos documentos acima e o discurso informal das entrevistas, tornou-se possível produzir, elaborar e fornecer informações sobre o assunto, permitindo fazer de várias formas a mesma pergunta relacionada às características das pragas que atacam a produção de soja. Essas perguntas estão sendo validadas por meio de um analisador léxico de domínio da agronomia para ser usado no ensino de pragas na cultura de soja.

Tal analisador dará, por exemplo, além da categoria morfológica da praga, seu nome científico, suas características biológicas, seu comportamento, os danos causados, sua localização na soja, os métodos de controle de danos e uma literatura disponível 
sobre cada uma das pragas. Para isso está sendo utilizada a tabela de símbolos recomendada por Moreno (2017).

Tendo em vista que o objetivo principal deste trabalho é auxiliar os estudantes da área da agronomia sobre o diagnóstico de pragas na cultura da soja, foi permitida a elaboração de questões possíveis de serem feitas pelo agricultor para promover consultas ricas, as quais são descritas abaixo:

- Qual o dano da praga Percevejo-marrom-da-Soja?

- Qual estado tem maior incidência da praga Formiga cortadeira?

- Qual praga ataca mais no estado do Paraná?

- Há quanto tempo surgiu a praga Larva-Angorá na sua lavoura?

- Alguém da mesma região teve a lavoura atingida pela mesma praga?

- Como controlar a praga Cochonilha-da-Raiz?

- Qual produto posso utilizar para controlar a praga Lagarta-cabeça-de-fósforo?

- Como identifico a praga Coró-da-Soja em minha lavoura?

- A praga na lavoura é recente?

- Está utilizando algum inseticida?

- É a primeira vez que a Mosca-branca aparece na lavoura?

- Qual a diferença entre o Percevejo-verde e o Percevejo-de-barriga-verde?

- Qual é o ciclo biológico da praga Mosca-Branca?

- Qual a origem da praga Tamanduá-da-Soja?

- Que danos são causados nas plantas de soja?

- Descreva o comportamento do Percevejo-castanho.

\section{I) Pergunta analisada}

a. Frase: "Qual é o ciclo biológico da praga Tamanduá-da-Soja?"

b. Padrão: Pronome relativo + verbo $[$ ser $]+$ artigo + substantivo comum + adjetivo + preposição + substantivo comum + substantivo composto.

\section{II) Resposta completa}

a. Frase: "Os ácaros fitófagos da soja podem ser naturalmente controlados em situações de chuvas intensas e periodos prolongados de umidade relativa elevada, especialmente porque essas condições propiciam o desenvolvimento de fungos entomopatogênicos e outros agentes de controle biológico natural no agroecossistema. Inseticidas organosfosforados, como metamidofós, profenofós, dimetoato, endosulfam e clorpirifós são sugeridos para o controle de ácaros na cultura da soja."

Questões como essa foram catalogadas e cada palavra individualmente foi classificada no dicionário de acordo com a categoria morfológica para que se possa formar um dicionário de bases, o qual será utilizado na análise léxico-morfológica das palavras.

Um protótipo de consulta por meio do framework pode ser visualizado na Figura 4, no qual é possível inserir uma pergunta em linguagem natural e obter uma resposta com 
base nos dados das pragas. Este protótipo ainda não está disponível, sendo necessária ainda uma análise sobre a usabilidade do sistema.

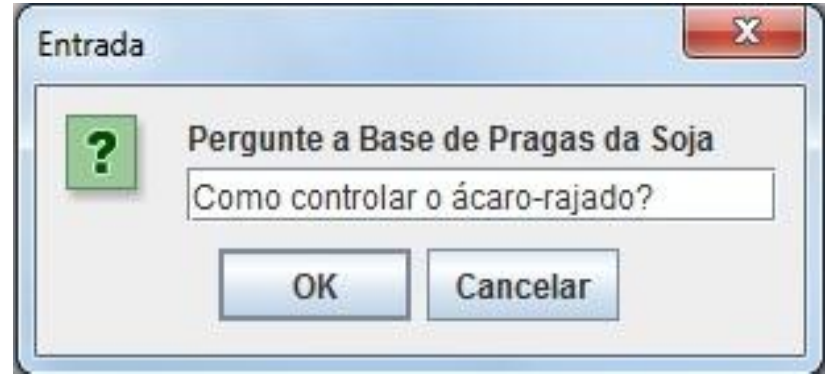

Figura 4 - Protótipo de Interface de Consulta na Base de Dados das Pragas

Respostas para essas consultas podem ser visualizadas, como na Figura 5.

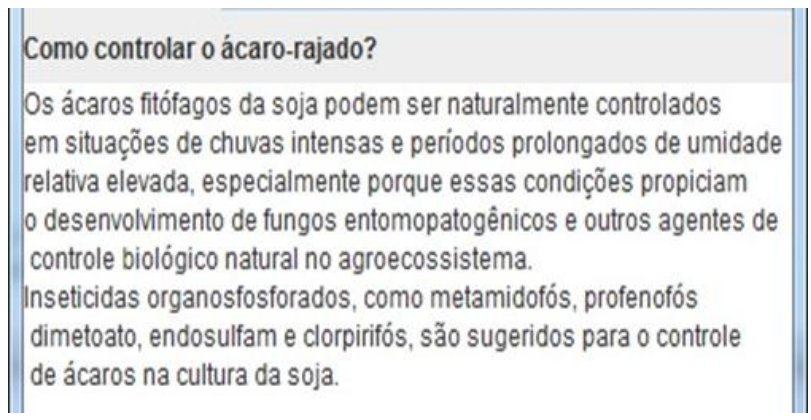

Figura 5 - Resposta de Consulta a uma pergunta sobre uma praga

Uma Gramática Livre de Contexto (GLC), baseada em Barbosa (1998), está sendo usada para gerar a linguagem agronômica. No referido trabalho é transformada essa GLC em uma Gramática Livre de Contexto Lexicalizada (GLCL), uma vez que a lexicalização é importante [Schabes e Waters 1993] do ponto de vista computacional, porque as gramáticas lexicalizadas podem muitas vezes ser analisadas mais eficientemente que as não-lexicalizados. Operações de substituição e adjunção como mostradas em Barbosa (1998) também podem ser usadas no domínio da agricultura.

Informalmente falando, essa gramática consiste, segundo Barbosa (1998), de dois conjuntos de árvores: as árvores iniciais, as quais são combinadas por substituição (Figura 6) e árvores auxiliares, as quais são combinadas por adjunções (Figuras 7 e 8). Quando as árvores são combinadas por essas operações, as representações semânticas são combinadas Uma GLCL é lexicalizada porque toda árvore inicial e auxiliar necessita conter uma palavra do dicionário em sua fronteira.

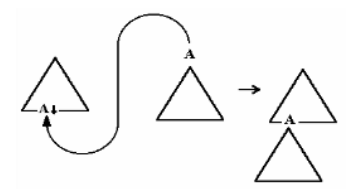

Figura 6 - Operação de Substituição na GLCL 


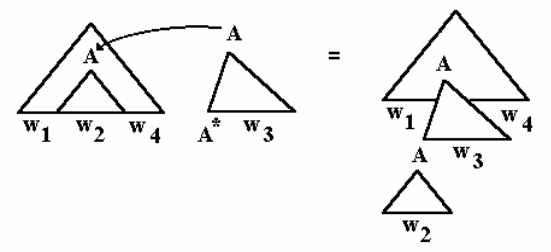

Figura 7 - Operação de Adjunção Recursiva à Esquerda na GLCL

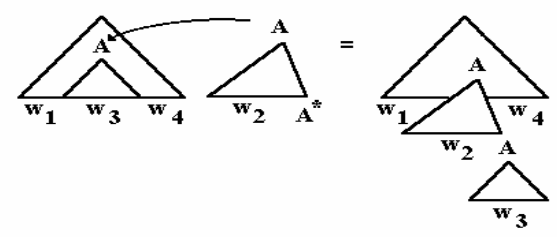

Figura 8 - Operação de Adjunção Recursiva à Direita na GLCL

A derivação nas GLCLs deve iniciar com uma árvore inicial rotulada em $S$. Depois disso, essa árvore pode ser repetidamente estendida usando-se substituição e adjunção. Uma derivação é completa quando todo nó na fronteira é rotulado com um símbolo terminal formando uma árvore sintática com categorias semânticas do nosso domínio da agricultura como, por exemplo, mostrada na Figura 8.

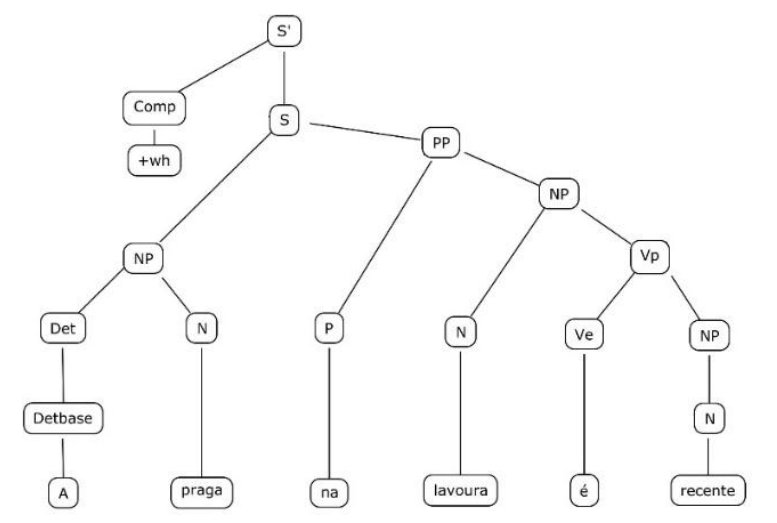

Figura 9 - Substituição em A praga na lavoura é recente

O marcador + wh em Comp indica um caso de pergunta interrogativa, como é o caso de A praga na lavoura é recente? Caso fosse uma pergunta imperativa como Descreva o comportamento do Percevejo-castanho, haveria a presença de $-w h$.

\section{Considerações Finais}

Foi exposto neste trabalho uma proposta de implementação de um sistema em Linguagem Natural para Banco de Dados desenvolvido para comprovação do modelo proposto. Ainda que não se encontre completo o dicionário, boa parte das pragas mais conhecidas na soja foi cadastrada, totalizando 78 pragas. Mais pragas ainda serão adicionadas à base de dados.

Posteriormente, para este trabalho, será feita a validação da gramática da linguagem de consulta apresentada em Barbosa (1998) e Barbosa (2004) para que possam ser realizadas as fases de análise sintática e semântica das frases da agronomia em linguagem natural. Sugere-se também o uso de famílias de árvores, como mostradas em Barbosa (2004), para reduzir a busca de frases válidas semanticamente dizendo. A 
intenção final é fazer um chatbot, que responderá a perguntas de estudantes de agronomia ou agrônomos sobre as pragas na cultura da soja. O uso de um chatbot tem a vantagem de poder utilizar da própria língua natural para realizar consultas em uma base de dados, por meio, por exemplo, de um smartphone.

Pretende-se ainda descrever mais consultas na base de dados da agricultura, mas para isso faz-se necessário previamente mais entrevistas com os agricultores para verificar se há mais dialetos nesse domínio para que sejam abarcadas no dicionário. É preciso validar se as regras gramaticais desse público são as mesmas da norma culta ou não para saber se há a necessidade de acrescentar mais regras à gramática proposta.

\section{Referências}

Agosti, C. (2003). Interface em Linguagem Natural para Banco de Dados: uma abordagem prática. 2003. Florianópolis: PPGCC da UFSC. 203p. Dissertação de Mestrado.

Agrolink. (2015). Pragas causam perdas de até $R \$ 55$ bilhões à agricultura no Brasil. https://www.agrolink.com.br/noticias/pragas-causam-perdas-de-ate-r--55-bilhoes-aagricultura-no-brasil_220429.html, acesso em: 07 mar. 2019.

Ávila, C. J. (2017). Pragas da soja e seu controle. https://pragas.cpao.embrapa.br, acesso em: 12 set. 2018.

Barbosa, C. R. S. C. de. (1997). Interfaces em Linguagem Natural para Banco de Dados. Porto Alegre: CPGCC da UFRGS. 165f. (TI-640)

(1998). Gramática para Consultas Radiológicas em Língua Portuguesa. Porto Alegre: CPGCC da UFRGS. 143f. Dissertação de Mestrado.

(2004). Técnicas de Parsing para Gramática Livre de Contexto Lexicalizada da Língua Portuguesa. São José dos Campos: CPG de Engenharia e Computação do ITA. 171f. Tese de Doutorado.

Carvalho, M. M., Bueno, R. C. O. F., Carvalho, L. C., Favoreto, A. L. e Godoy, A. L. (2013). Potencial do controle biológico para o controle de Pseudoplusia includens (Walker, 1857) e Anticarsia gemmatalis Hubner, 1818 (Lepidoptera: Noctuidae) em soja. Enciclopédia Biosfera, v. 9, n. 17, p. 2049-2063. http://hdl.handle.net/ 11449/137603, acesso em: 14 set. 2018.

Dale, R. (2010). Classical approaches to natural language processing. In Handbook of Natural Language Processing. Chapman and Hall/CRC, 2nd edition.

Dias-da-Silva, B. C., Montilha, G., Rino, L. H. M., Specia, L., Nunes, M. G. V., Oliveira Junior, O. N., Martins, R. T. e Pardo, T. A. S. (2007). Introdução ao Processamento das Línguas Naturais e Algumas Aplicações. Série de Relatórios do Núcleo Interinstitucional de Linguística Computacional. ICMC-USP, São Carlos. (NILC-TR-07-10)

Di Felippo, A. and Dias da Silva, B. C. (2006). Dos olhares sobre o léxico: diferenças e semelhanças. 1 ed. Araraquara: Laboratório Editorial da FCL da UNESP, v.09, p. 169-185.

EMBRAPA. (2018). Soja. https://www.embrapa.br/soja/publicacoes, acesso em: 19 set. 2018. 
Franco, J. R. (2017). Método computacional para identificação do fungo Cercospora Kikuchii em sementes de soja. Ponta Grossa: PPGCA da UEPG. Dissertação de Mestrado. 111f.

Goddard, C. and Schalley, A. C. (2010). Semantic analysis. In Handbook of Natural Language Processing. Chapman and Hall/CRC, 2nd edition.

$\mathrm{Gu}_{2}$ Y. and T, Jing. (2011). The IOT research in supply chain management of fresh agricultural products. In Proceedings of IEEE 2nd International Conference on Artificial Intelligence, Management Science and Electronic Commerce, Dengleng.

Hiraoka, E. e Jacopini, V. (2018). O papel da tecnologia na evolução da agricultura. http://www.sna.agr.br/o-papel-da-tecnologia-na-evolucao-da-agricultura, acesso em: 12 nov. 2018.

Manaris, B. Z. and Slator, B. M. (1996). Interactive Natural Language Processing: Building on Success. IEEE Computer, New York, 29(7), 28-32.

Moreira, H. J. C. e Aragão, F. D. (2009). Manual de Pragas da Soja. Campinas: FMC Agricultural Products. 144p.

Moreno, F. C., Manfio, E. R., Barbosa, C. R. S. C. e Brancher, J. D. (2015). Tical: Chatbot sobre o Atlas Linguístico do Brasil no WhatsApp. In XXVI Simpósio Brasileiro de Informática na Educação, Maceió. 279-288p.

Moreno, F. C. (2017). Visual TaHs: Ferramenta para analisar a eficácia de buscas das funções hash em um Léxico para Lingua Natural. Londrina: PPGCC da UEL. 202f. Dissertação de Mestrado.

Muller, D. N. (2003). Processamento de Linguagem Natural. Universidade Federal do Rio Grande do Sul. Technical Report.

Rocha, I. A. A. e Sartin, M. A. (2018). Pré processamento e segmentação de imagens de folhas de soja com base na visão computacional. In: Workshop de Tecnologias Emergentes em Computação, Sinop. https//www.even3.com.br/anais/wtec/95933pre-processamento-e-segmentacao-de-imagens-de-folhas-de-soja-com-base-navisao-computacional, acesso em: 16 nov. 2018.

Rosa, R. P. (2018). Método de classificação de pragas por meio de rede neural convolucional profunda. Ponta Grossa: PPGCA da UEPG. 99f. Dissertação de Mestrado.

Santos, O. S. (1995). A Cultura da Soja 1. Rio Grande do Sul-Santa Catarina-Paraná. 2. ed. São Paulo: Globo.

Schabes, Y. and Waters, R. C. (1993). Lexicalized Context-Free Grammar: A CubicTime Parsable, Lexicalized Normal Form For Context Free Grammar that preserves Tree Structure. Broadway, Cambridge: Mitsubishi Electric Research Laboratories. Technical Report 93- 04. June. 30p.

Silva, J. A. da. (2018). Ontologia na rastreabilidade de dados agrícolas. Ponta Grossa: PPGCA da UEPG. 74 f. Dissertação de Mestrado.

Sosa-Gomez, D. R., Corrêa-Ferreira, B. S., Hoffman-Campo, C. B., Corso, I. C., Oliveira, L. J., Moscardi, F., Panizzi, A. R., Bueno, A. F., Hirose, E. e Roggia, S. 
(2010). Manual de identificação de insetos e outros invertebrados da cultura da soja. Londrina, PR: Embrapa Soja (Documentos 269). 